\title{
Robot-assisted partial nephrectomy in contemporary practice
}

\section{Youssef S. Tanagho*, Sam B. Bhayani and Robert S. Figenshau}

Division of Urologic Surgery, Washington University School of Medicine, St. Louis, MO, USA

Edited by:

Brian Lane, Spectrum Health, USA

Reviewed by:

Jianbo Li, Cleveland Clinic, USA

Brock O'Neil, University of Utah, USA

*Correspondence:

Youssef S. Tanagho, Division of

Urologic Surgery, Washington

University School of Medicine

660 South Euclid Avenue, St. Louis, MO 63110, USA.

e-mail: tanaghoy@wudosis.wustl.edu

Laparoscopic renal surgery is associated with reduced blood loss, shorter hospital stay, enhanced cosmesis, and more rapid convalescence relative to open renal surgery. Laparoscopic partial nephrectomy (LPN) is a minimally invasive, nephron-sparing alternative to laparoscopic radical nephrectomy (RN) for the management of small renal masses. While offering similar oncological outcomes to laparoscopic RN, the technical challenges and prolonged learning curve associated with LPN limit its wider dissemination. Robot-assisted partial nephrectomy (RAPN), although still an evolving procedure with no long-term data, has emerged as a viable alternative to LPN, with favorable preliminary outcomes. This article provides an overview of the role of RAPN in the management of renal cell carcinoma. The clinical indications and principles of surgical technique for this procedure are discussed. The oncological, renal functional, and perioperative outcomes of RAPN are also evaluated, as are complication rates.

Keywords: robotic partial nephrectomy, robot-assisted partial nephrectomy, robotic surgery, minimally invasive surgical procedures, renal cell carcinoma, partial nephrectomy, outcomes assessment

\section{INTRODUCTION}

While the gold standard treatment of renal tumors was previously radical nephrectomy $(\mathrm{RN})$, investigation over the past decade has demonstrated the surgical feasibility and equivalent oncologic efficacy of partial nephrectomy (PN) for the management of small renal masses (SRM; Fergany et al., 2000). With mounting evidence indicating that overtreatment of renal masses with $\mathrm{RN}$ is associated with increased risk of chronic renal insufficiency, cardiovascular events, and premature deaths (Go et al., 2004; Thompson et al., 2008; Weight et al., 2010), the American Urological Association guidelines now explicitly endorse $\mathrm{PN}$ as the standard of care for managing T1a renal tumors and as an alternative treatment option for T1b tumors (American Urological Association Education and Research, 2009). Reflecting this paradigm shift, PN utilization has increased considerably at many centers of excellence over the past decade, approaching $90 \%$ for T1a tumors at some centers (Thompson et al., 2009).

With the rapid dissemination of minimally invasive technology within the urologic community, laparoscopic PN (LPN) and, more recently, robot-assisted PN (RAPN) have emerged as viable alternatives to open PN (OPN) for the management of suspected renal malignancy. The long-term oncological and functional outcomes of LPN are similar to those of OPN (Gill et al., 2007; Lane and Gill, 2010), with the potential benefits of reduced blood loss (estimated blood loss, EBL), shorter hospital stay (length of hospital stay, LOS), superior cosmesis, and more rapid convalescence. However, LPN remains technically demanding, necessitating

\footnotetext{
Abbreviations: EBL, estimated blood loss; eGFR, estimated glomerular filtration rate; LOS, length of hospital stay; LPN, laparoscopic partial nephrectomy; NSS nephron-sparing surgery; OPN, open partial nephrectomy; PN, partial nephrectomy; PSM, positive surgical margin; RAPN, robot-assisted partial nephrectomy; $\mathrm{RN}$, radical nephrectomy; SRM, small renal masses; WIT, warm ischemia time.
}

substantial technical expertise to achieve adequate tumor resection and renorrhaphy while minimizing ischemia times. Despite the development of novel techniques to facilitate LPN, the protracted learning curve associated with this procedure has hindered its dissemination into general practices in the U.S. and may, indeed, contribute to the underutilization of PN (Hollenback et al., 2006).

Among its potential advantages, robotic technology offers high-definition three-dimensional visualization, a broad range of wristed-instrument motion, and scaling of surgeon movements. RAPN appears to have a shorter learning curve than LPN (Mottrie et al., 2010) and, accordingly, may facilitate and promote the utilization of minimally invasive nephron-sparing surgery (NSS). We review the technique and outcomes of RAPN, assessing its current role and future prospects for the management of renal masses.

\section{SET UP}

For the inexperienced robotic renal surgeon, judicious patient selection is critical. The lack of haptic feedback and reliance on the bedside assistant can present challenges unique to RAPN. Patients ideally suited for initial RAPN procedures include those with nonhilar, exophytic Tla lesions, uncomplicated vascular anatomy, and a normal contralateral kidney.

\section{EQUIPMENT}

Robot-assisted partial nephrectomy is performed using the da Vinci surgical system (Intuitive Surgical Inc., Sunnyvale, CA, USA). Three robotic instruments are frequently used during RAPN - the Monopolar Curved Scissors in the dominant hand and ProGrasp forceps in the non-dominant hand; these are exchanged for robotic needle drivers during renorrhaphy. The ProGrasp's blunt tips are suited for dissection of vessels and tumor and can be used to apply robotic bulldog clamps (Scanlan International, 
St. Paul, MN, USA) and manipulate the robotic ultrasound probe, if needed. The PK (PlasmaKinetic) dissecting forceps, Maryland bipolar forceps, and robotic hook are other potentially useful instruments, which can aid in precise dissection and cauterization of small vessels. The scrubbed assistant employs conventional laparoscopic instruments to provide suction and countertraction via the assistant port. The fourth arm can be used with a retracting device to improve exposure, although instrument collisions may increase with its use, especially in patients with small torsos.

\section{PATIENT POSITIONING AND PORT PLACEMENT}

Patients undergoing transperitoneal RAPN are placed in a $75^{\circ}$ modified flank/lateral decubitus position with the pathologic side up. A $90^{\circ}$ full flank/lateral decubitus position is used for retroperitoneal RAPN (Gettman et al., 2004; Dulabon and Stifelman, 2011; White et al., 2011).

The transperitoneal approach is the most commonly used approach for RAPN. The most widely utilized transperitoneal camera/trocar configuration places the camera medial and superior to the umbilicus; a $30^{\circ}$ downward-angled lens is used. Two $8-\mathrm{mm}$ trocars for the robotic arms are placed just cephalad of the anterior superior iliac spine and inferior to the costal margin in the mid-axillary line. A $12-\mathrm{mm}$ assistant port is placed in the midline in either the upper or lower quadrant, depending on tumor location and surgeon preference. If a fourth robotic arm is used, an additional $8-\mathrm{mm}$ trocar is placed laterally, triangulated between the two other robotic trocars (Cabello et al., 2009; Kavoussi et al., 2011). The robot is docked posterior to the patient.

Access for retroperitoneal RAPN is obtained through a $1.2-\mathrm{cm}$ skin incision just inferior to the tip of the 12 th rib. The flank muscle fibers and thoracolumbar fascia are bluntly split, and the surgeon's fingertip creates a potential space between the psoas muscle and Gerota's fascia; this space is further expanded by injection of $800 \mathrm{~mL}$ of air into the retroperitoneum through a balloon dilator. The camera port is then placed at the site of the balloon dilator. Generally, only two additional working ports, triangulated with the camera at an obtuse angle to reduce instrument collisions, are required. If a fourth robotic arm is used, the peritoneum is pushed medial to the belly of the rectus in order to expand the retroperitoneal workspace, and the fourth arm is placed anteriorly in that location. An assistant port is placed in the lower quadrant (Rogers, 2009; Dulabon and Stifelman, 2011). The robot is docked anterior to the patient.

\section{SURGERY}

Depending on tumor location, patient surgical history, and surgeon preference, a transperitoneal or retroperitoneal approach is selected. While safe and effective in experienced hands, the retroperitoneal approach is potentially more challenging due to its confined workspace and relatively fewer anatomic landmarks (Weizer et al., 2011).

\section{LESION EXCISION}

Following tumor exposure, its precise borders are delineated, often under intraoperative ultrasound guidance. The newly developed robot-controlled ultrasound probe (Aloka, Tokyo, Japan) allows full surgeon control of intraoperative imaging. TilePro software integration, included in newer robotic platforms, allows for real-time picture-on-picture display of radiographic images on the console screen, thus facilitating the mapping out of the dissection.

Traditionally, renal hilar vessels are clamped - either individually (starting with the artery) using laparoscopic bulldog clamps or en bloc using a laparoscopic Satinsky clamp - prior to tumor excision; the latter requires placement of a dedicated port. Recently developed "robotic bulldog clamps" provide the surgeon additional autonomy, in lieu of having to relegate the delicate task of hilar occlusion to the assistant. The tumor is excised sharply with a rim of normal renal parenchyma. The assistant applies countertraction with the suction device to enhance visualization during tumor excision.

\section{REPAIR}

Using robotic needle drivers, renorrhaphy is traditionally performed in two layers. A deep-layer closure of the resection bed, which includes repair of large blood vessels and collecting system defects, is first performed with a poliglecaprone 25 or polyglactin suture in a running fashion. This is followed by an outer-layer closure of the renal capsule, performed using larger absorbable sutures and needles. The Washington University technique of "sliding-clip renorrhaphy" - widely adopted as a preferable alternative to the traditional tied-suture renorrhaphy - relies upon the use of Weck Hem-o-Lok clips, placed on either side of the defect and then slid into place by the surgeon, to exert tension upon the repair. The Hem-o-Lok clips are generally reinforced with LapraTy clips to prevent backsliding of the clips. This technique is ideally suited for RAPN, as the robotic instrumentation affords the surgeon the requisite precision in dictating the degree of tension placed on the repair, effectively eliminating the need for placement of surgical bolsters in the renal defect to achieve tight closure (Benway et al., 2009c).

\section{TECHNIQUES TO MINIMIZE WARM ISCHEMIA}

As minimally invasive techniques for achieving renal hypothermia during renal hilar clamping have failed to gain widespread clinical application, RAPN is generally performed under conditions of "warm ischemia." In recognition of the potential adverse effect that even limited warm ischemia time (WIT) may have on kidney function (Lane et al., 2008; Becker et al., 2009; Thompson et al., 2010), multiple investigators have compared WIT during RAPN vs. LPN. While several earlier studies found no significant difference in WIT between RAPN and LPN (Caruso et al., 2006; Aron et al., 2008; Deane et al., 2008), a recent study comparing 129 patients who underwent RAPN and 118 patients who underwent LPN demonstrated significantly reduced WIT in the RAPN group (19.7 vs. 28.4 min, $p<0.001$ ); RAPN maintained consistently shorter WIT than LPN in patients with "complex" renal tumors (25.9 vs. $36.7 \mathrm{~min}, p<0.001$; Benway et al., 2009b). Other smaller comparative series of RAPN and LPN have also demonstrated a reduction in WIT with RAPN (Wang and Bhayani, 2008; Williams etal., 2011). Importantly, individual surgeon experience significantly impacts WIT independent of surgical technique. Nonetheless, preliminary investigation suggests that RAPN may not require as long a learning curve as LPN to achieve a reasonable 
WIT (Mottrie et al., 2010). WIT in multiple RAPN series are presented in Table 1 (Gettman et al., 2004; Bhayani and Das, 2008; Rogers et al., 2008; Benway et al., 2010; Mottrie et al., 2010; Patel et al., 2010; Scoll et al., 2010; Dulabon et al., 2011; Lorenzo et al., 2011; Naeem et al., 2011; Petros et al., 2011; Simhan et al., 2012), and studies comparing WIT during RAPN vs. LPN are outlined in Table 2 (Caruso et al., 2006; Aron et al., 2008; Deane et al., 2008; Wang and Bhayani, 2008; Benway et al., 2009b; Haber et al., 2010; Williams et al., 2011).

By simplifying the renorrhaphy, while minimizing reliance on the surgical assistant, the sliding-clip technique, as described above, has been shown to substantially reduce WIT during RAPN, compared to tied-suture renorrhaphy (Benway etal., 2009c). Precluding the need for additional "anchoring" LapraTy clips, the more recent application of barbed suture to facilitate tight parenchymal closure during sliding-clip renorrhaphy may further decrease WIT (Sammon et al., 2011; Sukumar and Rogers, 2011).

Other variations in surgical technique previously described for OPN and LPN have also been applied to RAPN in an effort to minimize or eliminate WIT. Some have adopted an "early unclamping technique," clamping the renal hilum only during tumor resection and while suturing large vessels/collecting system at the resection base (San Francisco et al., 2011). Others have performed "selective renal parenchymal clamping," clamping regional blood vessels only in the area of planned excision (Figenshau, 2005; Benway et al., 2009a). More recently, some have performed RAPN without any clamping of the renal hilum, suggesting that off-clamp RAPN can be safely performed in carefully selected patients (Wu et al., 2010; Tanagho et al., 2012a,b). Our initial experience with offclamp RAPN at Washington University demonstrates favorable renal functional outcomes with this technique (Tanagho et al., 2012a,b). Nevertheless, several studies have failed to demonstrate long-term improvement in renal functional outcomes after any modification of clamping technique or in the absence of renal hilar clamping (Bhayani et al., 2004; Foyil et al., 2008; Becker et al., 2009). Further studies will be needed to establish the efficacy and reaffirm the safety of these surgical approaches.

\section{COMPLICATIONS}

Early series of RAPN reported complication rates ranging from 0 to $20 \%$ (Cha et al., 2011). A contemporary study of 886 consecutive cases of RAPN performed at five U.S. centers reported an overall complication rate of $15.6 \%$, with intraoperative and postoperative complication rates of 2.6 and $13.0 \%$, respectively. Postoperative complications were classified as Clavien grade I-II in $77.0 \%$ of cases and grade III-IV in $23.0 \%$. RAPN was converted to OPN or LPN in $0.2 \%$ of patients and to $\mathrm{RN}$ in $0.5 \%$ of patients. There were no deaths (Tanagho et al., 2012c). Table 1 summarizes complication rates of various RAPN series (Gettman et al., 2004; Bhayani and Das, 2008; Rogers et al., 2008; Benway et al., 2010; Mottrie et al., 2010; Patel et al., 2010; Scoll et al., 2010; Dulabon et al., 2011; Lorenzo et al., 2011; Naeem et al., 2011; Petros et al., 2011; Simhan et al., 2012).

Reported complication rates of RAPN are comparable to those seen in OPN and LPN. For example, Gill et al. (2007) reported complications in 13.7 and $18.6 \%$ of patients undergoing OPN and LPN, respectively. A study by Simhan et al. (2012) comparing outcomes of RAPN vs. OPN in 281 patients demonstrated similar major and minor complication rates in the two groups. Benway etal. (2009b) compared 129 patients who underwent RAPN with 118 patients who underwent LPN and found no

Table 1 | Contemporary robotic partial nephrectomy case series.

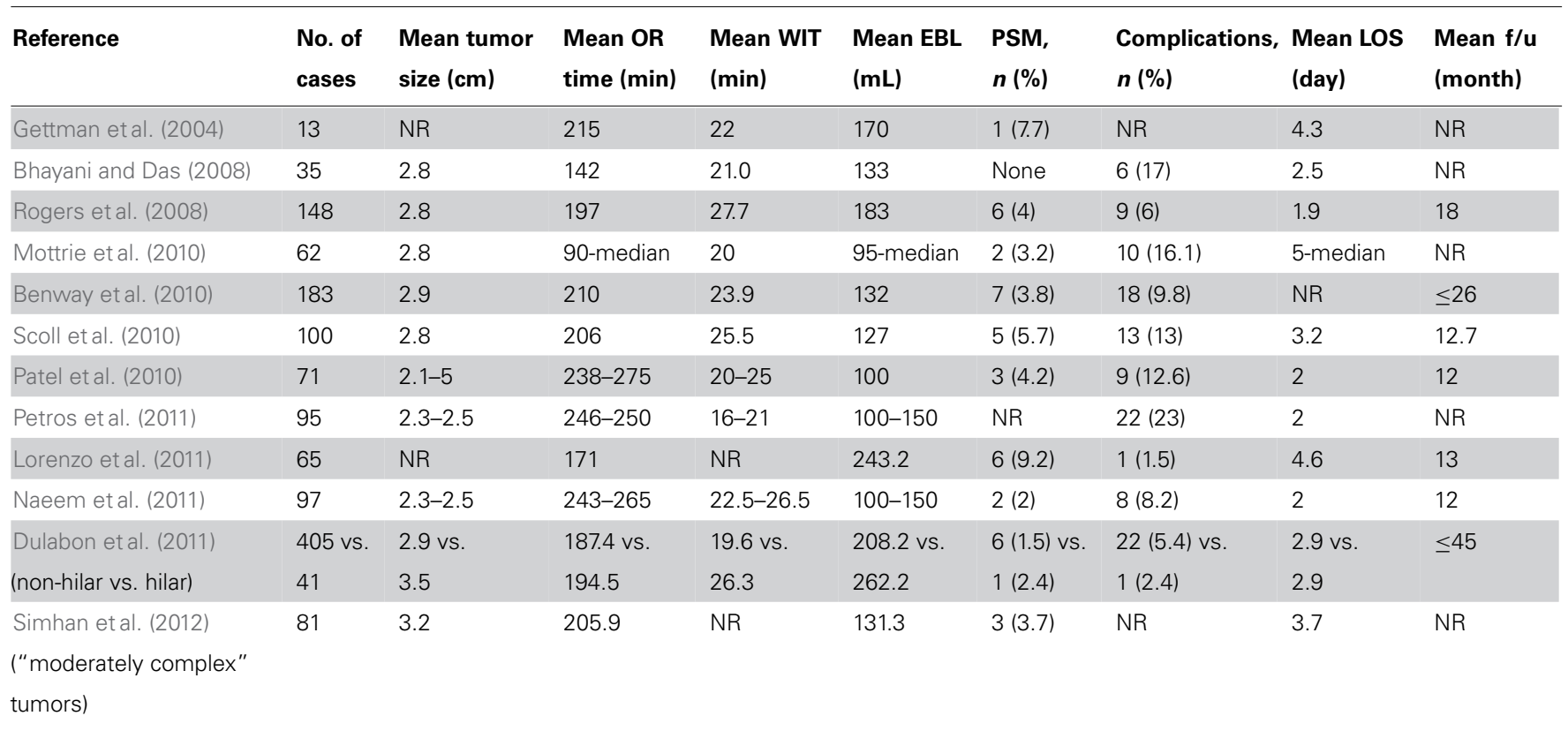

WIT, warm ischemia time, EBL; estimated blood loss; LOS, length of stay; PSM, positive surgical margin; f/u, follow-up; NR, not reported. 
Table 2 | Series comparing RAPN vs. LPN.

\begin{tabular}{|c|c|c|c|c|c|c|c|c|c|}
\hline Reference & $\begin{array}{l}\text { No. of } \\
\text { cases }\end{array}$ & $\begin{array}{l}\text { Mean tumor } \\
\text { size }(\mathrm{cm})\end{array}$ & $\begin{array}{l}r \text { Mean OR } \\
\text { time (min) }\end{array}$ & $\begin{array}{l}\text { Mean WIT } \\
(\min )\end{array}$ & $\begin{array}{l}\text { Mean EBL } \\
(\mathrm{mL})\end{array}$ & $\begin{array}{l}\text { PSM, } \\
n(\%)\end{array}$ & $\begin{array}{l}\text { Complications, } \\
n(\%)\end{array}$ & $\begin{array}{l}\text { Mean LOS } \\
\text { (day) }\end{array}$ & $\begin{array}{l}\text { Mean f/u } \\
\text { (month) }\end{array}$ \\
\hline Caruso et al. (2006) & RAPN: 10 & RAPN: 2.0 & RAPN: 279 & RAPN: 26.4 & RAPN: 240 & RAPN: 0 (0) & RAPN: 1 (10) & RAPN: 2.6 & RAPN: NR \\
\hline \multirow[t]{2}{*}{ Aron et al. (2008) } & RAPN: 12 & RAPN: 2.4 & RAPN: 242 & RAPN: 23 & RAPN: 329 & RAPN: NR & RAPN: NR & RAPN: 4.7 & RAPN: 7.4 \\
\hline & LPN: 12 & LPN: 2.9 & LPN: 256 & LPN: 22 & LPN: 300 & LPN: NR & LPN: NR & LPN: 4.4 & LPN: 8.5 \\
\hline & LPN: 11 & LPN: 2.3 & LPN: 290 & LPN: 35.3 & LPN: 198 & LPN: 1 (9.1) & LPN: 1 (9.1) & LPN: 3.1 & LPN: 4.5 \\
\hline \multirow[t]{2}{*}{ Benway et al. (2009b) } & RAPN: 129 & RAPN: 2.9 & RAPN: 189 & RAPN: 19.7 & RAPN: 155 & RAPN: 5 (3.9) & RAPN: 11 (8.5) & RAPN: 2.4 & RAPN: NR \\
\hline & LPN: 118 & LPN: 2.6 & LPN: 174 & LPN: 28.4 & LPN: 196 & LPN: 1 (0.8) & LPN: 12 (10.2) & LPN: 2.7 & LPN: NR \\
\hline \multirow[t]{2}{*}{ Wang and Bhayani (2008) } & RAPN: 40 & RAPN: 2.5 & RAPN: 140 & RAPN: 19 & RAPN: 137 & RAPN: 1 (2.5) & RAPN: $8(20)$ & RAPN: 2.5 & RAPN: NR \\
\hline & LPN: 62 & LPN: 2.4 & LPN: 156 & LPN: 25 & LPN: 173 & LPN: 1 (1.6) & LPN: 9 (14.5) & LPN: 2.9 & LPN: NR \\
\hline & LPN: 59 & LPN: 3.1 & LPN: 221 & LPN: 28.0 & LPN: 146 & LPN: 7 (11.9) & LPN: 12 (20.3) & LPN: 2.7 & LPN: NR \\
\hline
\end{tabular}

RAPN, robot-assisted partial nephrectomy; LPN, laparoscopic partial nephrectomy; WIT, warm ischemia time; EBL, estimated blood loss; LOS, length of stay; PSM, positive surgical margin; f/u, follow-up; NR, not reported.

significant differences in complication rates. Series comparing complication rates of RAPN and LPN are presented in Table 2 (Caruso et al., 2006; Aron et al., 2008; Deane et al., 2008; Wang and Bhayani, 2008; Benway et al., 2009b; Haber et al., 2010; Williams et al., 2011).

In the following section, some of the more common and significant complications of RAPN are discussed.

\section{HEMORRHAGE \\ Intraoperative}

Tanagho et al.'s (2012c) multi-institutional analysis of RAPN complications demonstrated an intraoperative hemorrhage rate of $1.0 \%$. (Hemorrhage was defined as bleeding requiring blood transfusion or therapeutic intervention.) Parenchymal bleeding during tumor excision may result from inadequate hilar occlusion or unrecognized accessory vessels. Pulsatile bleeding of a typically larger volume indicates an arterial source, while a lower volume ooze suggests a venous source. In the case of arterial bleeding, controlled anesthetic blood pressure reduction may enhance visualization, and temporary removal of the venous clamp may reduce parenchymal congestion. En bloc hilar clamping with a Satinsky clamp or a long bulldog clamp is another option. Venous bleeding can often be mitigated by raising the pneumoperitoneum up to $18 \mathrm{mmHg}$. In contrast to the renal vein unclamping maneuver recommended for arterial bleeding, with venous bleeding, an additional clamp may be placed on the renal vein to minimize venous backflow. Inadequate sutured renal reconstruction may lead to bleeding after the renal hilum is unclamped. Direct pressure is immediately applied and the insufflation pressure increased while the renorrhaphy clips are re-tightened. Emergent open conversion or conversion to robotic total nephrectomy may be necessary for uncontrolled bleeding (Nepple et al., 2012).

\section{Postoperative}

In our multi-institutional analysis of RAPN complications, we reported a postoperative hemorrhage rate of $5.8 \%$ (Tanagho et al., 2012c). Published postoperative transfusion rates for RAPN range from 3 to $10 \%$, which are comparable to the 5.8 and $3.4 \%$ rates for LPN and OPN, respectively (Cha et al., 2011). Pseudoaneurysm or arteriovenous fistula formation may result in delayed postoperative hemorrhage, often presenting several weeks after discharge (Benway etal., 2009b). Angiography and embolization are indicated for persistent bleeding and/or hemodynamic instability.

\section{URINE LEAK}

Although initial urinary leak rates reported for RAPN ranged from 2 to 12.5\% (Cha et al., 2011), Tanagho et al.'s (2012c) more contemporary multi-center study of RAPN complications reported urine leakage (defined as urine extravasation identified radiographically or "persistently" increased drain fluid creatinine) in only $1.1 \%$ of cases; this rate is significantly lower than the 3.1 and $2.3 \%$ rates reported in LPN series (American Urological Association Education and Research, 2009; Cha etal., 2011). In the absence of obstruction distal to the leakage site, the majority of urine leaks will spontaneously resolve within several weeks. Retrograde placement of a ureteric stent may be indicated if conservative management fails (Meeks et al., 2008).

\section{RESULTS}

Taken together, single series (Table 1; Gettman et al., 2004; Bhayani and Das, 2008; Rogers et al., 2008; Benway et al., 2010; Mottrie et al., 2010; Patel et al., 2010; Scoll et al., 2010; Dulabon et al., 2011; Lorenzo et al., 2011; Naeem et al., 2011; Petros et al., 2011; Simhan et al., 2012) and comparative studies (Table 2; Caruso et al., 2006; 
Aron et al., 2008; Deane et al., 2008; Wang and Bhayani, 2008; Benway etal., 2009b; Haber etal., 2010; Williams et al., 2011) have demonstrated that RAPN can be performed safely and with acceptable oncological, functional, and perioperative outcomes. Nonetheless, carefully matched (ideally, randomized) comparisons of OPN, LPN, and RAPN with long-term follow-up are still required. The recent development of metrics for comparing renal mass complexity (e.g., R.E.N.A.L. nephrometry score, Kutikov and Uzzo, 2009; and PADUA score, Ficarra et al., 2009) may facilitate such comparisons.

\section{ONCOLOGIC OUTCOMES}

Because RAPN is a novel and maturing technique, positive surgical margin (PSM) rates have often been reported as a surrogate for oncological control. A review of contemporary RAPN series demonstrated a cumulative PSM rate of $2.7 \%$ (Benway and Bhayani, 2011), which is comparable to the 2.9 and $1.3 \%$ rates previously reported for LPN and OPN, respectively (Gill et al., 2007). PSM rates in various RAPN series are depicted in Table 1 (Gettman et al., 2004; Bhayani and Das, 2008; Rogers et al., 2008; Benway et al., 2010; Mottrie et al., 2010; Patel etal., 2010; Scoll et al., 2010; Dulabon et al., 2011; Lorenzo et al., 2011; Naeem et al., 2011; Petros et al., 2011; Simhan et al., 2012), while studies comparing PSM rates between RAPN and LPN are outlined in Table 2 (Caruso et al., 2006; Aron et al., 2008; Deane et al., 2008; Wang and Bhayani, 2008; Benway et al., 2009b; Haber et al., 2010; Williams et al., 2011).

Early and intermediate outcomes of RAPN show excellent oncological control (Kyllo et al., 2012). In fact, a review of modern large RAPN series encompassing $>1600$ patients demonstrated only seven recurrences, a rate of $<1 \%$ (Benway and Bhayani, 2011). Although these early reports are certainly encouraging, long-term data on RAPN are presently lacking. Long-term oncological outcomes from the largest series of LPN were recently reported and were comparable with those of OPN (Lane and Gill, 2010). Several recent studies have shown that the oncological outcomes of RAPN are equivalent to those of LPN in the short and intermediate term (Aron et al., 2008; Rogers et al., 2008; Wang and Bhayani, 2008; Patel et al., 2010; Dulabon et al., 2011).

\section{RENAL FUNCTIONAL OUTCOMES}

Overtreatment with $\mathrm{RN}$ has been linked to increased risk of chronic renal insufficiency and higher mortality (Huang et al., 2006; Thompson et al., 2008; Weight et al., 2010). A contemporary series of patients with unanticipated benign tumors demonstrated a 2.5-fold increased mortality 5 years following $\mathrm{RN}$, compared to PN (open or laparoscopic; Weight et al., 2010). The clear advantage of NSS in preserving renal function which numerous OPN and LPN series have shown has been similarly demonstrated in series of RAPN. Indeed, an international, multi-center study of 183 patients showed no significant postoperative change in estimated glomerular filtration rate (eGFR; 82.2 vs. $79.4 \mathrm{~mL} / \mathrm{min} / 1.73 \mathrm{~m}^{2}, p=0.74$ ) up to 26 months following RAPN (Benway et al., 2010). Simhan's study comparing outcomes of RAPN vs. OPN demonstrated similar percent changes in eGFR in the two groups (Simhan et al., 2012). Studies comparing renal functional outcomes of RAPN vs. RN and RAPN vs. LPN are still needed.

\section{PERIOPERATIVE OUTCOMES}

Early studies by Caruso, Deane, and Aron reported no significant differences in operative time or EBL between RAPN and LPN (Caruso et al., 2006; Aron et al., 2008; Deane et al., 2008). Similarly, in a matched cohort study of 150 patients undergoing RAPN or LPN, Haber et al. (2010) found no significant difference in operative time and LOS.

To the contrary, Benway et al. (2009b) reported a significant decrease in EBL (155 vs. $196 \mathrm{~mL}, p=0.03$ ) and LOS (2.4 vs. 2.7 days, $p<0.001)$ in patients undergoing RAPN vs. LPN, respectively. Wang and Bhayani (2008) also reported a shorter operative time ( 140 vs. $156 \mathrm{~min}, p=0.04$ ) and LOS (2.5 vs. 2.9 days, $p=0.03$ ) for RAPN in their series of 40 RAPNs and 62 LPNs. Table 1 depicts perioperative outcomes of various RAPN series (Gettman et al., 2004; Bhayani and Das, 2008; Rogers et al., 2008; Benway et al., 2010; Mottrie et al., 2010; Patel et al., 2010; Scoll et al., 2010; Dulabon et al., 2011; Lorenzo et al., 2011; Naeem et al., 2011; Petros et al., 2011; Simhan et al., 2012), while studies comparing perioperative outcomes of RAPN vs. LPN are summarized in Table 2 (Caruso et al., 2006; Aron et al., 2008; Deane et al., 2008; Wang and Bhayani, 2008; Benway et al., 2009b; Haber et al., 2010; Williams et al., 2011).

\section{CONCLUSION}

Despite the growing acknowledgment of elective PN as a feasible, oncologically sound, and less morbid treatment option for managing SRM, PN remains grossly underutilized, particularly in the community setting (Cooperberg et al., 2011). In the absence of adequate ancillary health services, the greater technical complexity and higher risk of vascular and urinary complications associated with PN (American Urological Association Education and Research, 2009) may dissuade general urologists from performing this procedure electively. Furthermore, given the potential advantages and popularization of minimally invasive surgery over open surgery, the advanced level of technical proficiency required to perform LPN may deter some surgeons from performing a minimally invasive nephron-sparing procedure in favor of the less technically demanding laparoscopic RN.

Surmounting some of the technical challenges associated with LPN (Deane et al., 2008; Mottrie et al., 2010), the emergence of RAPN as a minimally invasive nephron-sparing alternative to LPN may play a critical role in facilitating the wider dissemination of NSS into general practices. Indeed, with growing expertise in robotic surgery, RAPN has been offered to an increasing number of patients, including those with larger, endophytic, and central masses (Cha et al., 2011); moreover, studies have demonstrated that RAPN can, in fact, be performed safely and with acceptable outcomes for renal tumors of increasingly greater complexity (Rogers et al., 2008; Patel et al., 2010; Dulabon et al., 2011). Comparative studies of RAPN vs. LPN have also demonstrated favorable outcomes for RAPN (Caruso et al., 2006; Aron et al., 2008; Deane et al., 2008; Wang and Bhayani, 2008; Benway et al., 2009b; Haber et al., 2010; Williams et al., 2011). Nonetheless, long-term data on RAPN - essential for the full espousal of this technique - are presently lacking.

As a nascent procedure, the technique of RAPN continues to evolve. Further advances in minimizing WIT and facilitating the 
use of cold ischemia are expected. Technical innovation in robotic instrumentation may also enhance the technique of RAPN. For example, the development of systems such as TilePro, which enable picture-on-picture display of radiographic images on the console screen, may facilitate precise tumor dissection. The addition of the fourth robotic arm in the da Vinci S and Si systems decreases the surgeon's dependence on the bedside assistant during retraction, dissection, and reconstruction. Furthermore, with

\section{REFERENCES}

American Urological Association Education and Research (AUA). (2009). Guidelines for Management of the Clinical Stage I Renal Mass. Available at: www.auanet.org/content/ media/renalmass09.pdf

Aron, M., Koenig, P., Kaouk, J. H., Nquyen, M. M., Desai, M. M., and Gill, I. S. (2008). Robotic and laparoscopic partial nephrectomy: a matched-pair comparison from a high-volume center. BJU Int. 102, 86-92.

Becker, F., Van Poppel, H., Hakenberg, O. W., Stief, C., Gill, I., Guazzoni, G., et al. (2009). Assessing the impact of ischemia time during partial nephrectomy. Eur. Urol. 56, 625-634.

Benway, B. M., Baca, G., Bhayani, S. B., Das, N. A., Katz, M. D., Diaz, D. L., et al. (2009a). Selective versus nonselective arterial clamping during laparoscopic partial nephrectomy: impact upon renal function in the setting of a solitary kidney in a porcine model. J. Endourol. 23, 1127-1133.

Benway, B. M., Bhayni, S. B., Rogers, C. G., Dulabon, L. M., Patel, M. N., Lipkin, M., et al. (2009b). Robot-assisted partial nephrectomy versus laparoscopic partial nephrectomy for renal tumors: a multi-institutional analysis of peri-operative outcomes. J. Urol. $182,866-872$.

Benway, B. M., Wang, A. J., Cabello, J. M., and Bhayani, S. B. (2009c). Robotic partial nephrectomy with sliding-clip renorrhaphy: technique and outcomes. Eur. Urol. 55, 592-599.

Benway, B. M., and Bhayani, S. B. (2011). Surgical outcomes of robotassisted partial nephrectomy. BJU Int. 108, 955-961.

Benway, B. M., Bhayani, S. B., Rogers, C. G., Porter, J. R., Buffi, N. M., Figenshau, R. S., et al. (2010). Robotassisted partial nephrectomy: an international experience. Eur. Urol. 57, 815-820.

Bhayani, S. B., and Das, N. (2008). Robotic assisted laparoscopic partial nephrectomy for suspected renal cell carcinoma: retrospective review of surgical outcomes of 35 cases. BMC
Surg. 8:16. doi: 10.1186/1471-24828-16

Bhayani, S. B., Rha, K. H., Pinto, P. A., Ong, A. M., Allaf, M. E., Trock, B. J., et al. (2004). Laparoscopic partial nephrectomy: effect of warm ischemia time on serum creatinine. $J$. Urol. 172, 1264-1266.

Cabello, J. M., Bhayani, S. B., Figenshau, R. S., and Benway, B. M. (2009). Camera and trocar placement for robot-assisted radical and partial nephrectomy: which configuration provides optimal visualization and instrument mobility? J. Robot. Surg. 3, 155.

Caruso, R. P., Phillips, C. K., Kau, E., Taneja, S. S., and Stifelman, M. D. (2006). Robot-assisted laparoscopic partial nephrectomy: initial experience. J. Urol. 176, 36-39.

Cha, E. K., Lee, D. J., and Del Pizzo, J. J. (2011). Current status of robotic partial nephrectomy (RPN). BJU Int. 108, 935-941.

Cooperberg, M. R., Mallin, K., Kane, C. J., and Carroll, P. R. (2011). Treatment trends for stage I renal cell carcinoma. J. Urol. 186, 394-399.

Deane, L. A., Lee, H. J., Box, G. N., Melamud, O., Yee, D. S., Abraham, J. B., etal. (2008). Robotic versus standard laparoscopic partial/wedge nephrectomy: a comparison of intraoperative and perioperative results from a single institution. J. Endourol. 22, 947-952.

Dulabon, L. M., Kaouk, J. H., Haber, G. P., Berkman, D. S., Rogers, C. G., Petros, F., etal. (2011). Multiinstitutional analysis of robotic partial nephrectomy for hilar versus nonhilar lesions in 446 consecutive cases. Eur. Urol. 59, 325-330.

Dulabon, L. M., and Stifelman, M. D. (2011). "Laparoscopic and robotic partial nephrectomy," in Retroperitoneal Robotic and Laparoscopic Surgery, eds J. V. Joseph and H. R. Patel (London: SpringerVerlag), 61.

Fergany, A. F., Hafez, K. S., and Novick, A. C. (2000). Long-term results of nephron sparing surgery for localized renal cell carcinoma: 10-year followup. J. Urol. 163, 442-445.

Ficarra, V., Novara, G., Secco, S., Macchi, V., Porzionato, A., De Caro,

the introduction of the robotic ultrasound probe, robotic bulldog clamps, and barbed suture, the trend for maximizing the autonomy of the console surgeon during the critical steps of tumor identification, hilar clamping, and renorrhaphy is becoming increasingly apparent. The future design of robotic systems capable of providing tactile feedback to the surgeon may also contribute to the safety and efficacy of RAPN, particularly for the management of complex renal masses.

R., et al. (2009). Preoperative aspects and dimensions used for anatomical (PADU) classification of renal tumors in patients who are candidates for nephron-sparing surgery. Eur. Urol. 56, 786-793.

Figenshau, R. S. (2005). Laparoscopic partial nephrectomy with segmental renal vascular control. J. Endourol. 19(Suppl. 1), A257.

Foyil, K. V., Ames, C. D., Ferguson, G. G., Weld, K. J., Figenshau, R. S., Venkatesh, R., et al. (2008). Longterm changes in creatinine clearance after laparoscopic renal surgery. $J$. Am. Coll. Surg. 206, 511-515.

Gettman, M. T., Blute, M. L., Chow, G. K., Neururer, R., Bartsch, G. and Peschel, R. (2004). Roboticassisted laparoscopic partial nephrectomy: technique and initial clinical experience with DaVinci robotic system. Urology 64, 914-918.

Gill, I. S., Kavoussi, L. R., Lane, B. R., Blute, M. L., Babineau, D., Colombo, J. R. Jr., et al. (2007). Comparison of 1,800 laparoscopic and open partial nephrectomies for single renal tumors. J. Urol. 178, 41-46.

Go, A. S., Chertow, G. M., Fan, D., McCulloch, C. E., and Hsu, C. Y (2004). Chronic kidney disease and the risks of death, cardiovascular events, and hospitalization. N. Engl. J. Med. 351, 1296-1305.

Haber, G. P., White, W. M., Crouzet, S., White, M. A., Forest, S., Autorino, R. et al. (2010). Robotic versus laparoscopic partial nephrectomy: singlesurgeon matched cohort study of 150 patients. Urology 76, 754-758.

Hollenback, B. K., Taub, D. A., Miller D. C., Dunn, R. L., and Wei, J. T. (2006). National utilization trends of partial nephrectomy for renal cell carcinoma: a case of underutilization? Urology 67, 254.

Huang, W. C., Levey, A. S., Serio, A. M., Snyder, M., Vickers, A. J., Raj, G. V., et al. (2006). Chronic kidney disease after nephrectomy in patients with renal cortical tumors: a retrospective cohort study. Lancet Oncol. 7, 735-740.

Kavoussi, L. R., Schwartz, M. J., and Gill, I. S. (2011). "Laparoscopic surgery of the kidney," in: Campbell-Walsh Urology, 10th Edn, eds A. J. Wein,
L. R. Kavoussi, A. C. Novick, A. W. Partin, and C. A. Peters (Philadelphia: Saunders, Elsevier Inc.), Chapter 55. Kutikov, A., and Uzzo, R. G. (2009). The R.E.N.A.L. nephrometry score: a comprehensive standardized system for quantitating renal tumor size, location, and depth. J. Urol. 182, 844-853.

Kyllo, R., Tanagho, Y. S., Kaouk, J. H., Stifelman, M. D., Rogers, C. G., Hillyer, S. P., et al. (2012). Prospective multi-center study of oncologic outcomes of robot-assisted partial nephrectomy for pT1 renal cell carcinoma. BMC Urol. 12:11. doi: 10.1186/1471-2490-12-11

Lane, B. R., Babineau, D. C., Poggio, E. D., Weight, C. J., Larson, B. T., Gill, I. S., et al. (2008). Factors predicting renal functional outcome after partial nephrectomy. J. Urol. 180, 2363-2368.

Lane, B. R., and Gill, I. S. (2010). 7-year oncological outcomes after laparoscopic and open partial nephrectomy. J. Urol. 183, 473-479.

Lorenzo, E. I., Jeong, W., Oh, C. K., Chung, B. H., Choi, Y. D., and Rha, K. H. (2011). Robotics applied in laparoscopic kidney surgery. The Yonsei University experience of 127 cases. Urology 77, 114-118.

Meeks, J. J., Zhao, L. C., Navai, N., Perry, K. T. Jr., Nadler, R. B., et al. (2008). Risk factors and management of urine leaks after partial nephrectomy. J. Urol. 180, 2375-2378.

Mottrie, A., De Naeyer, G., Schatteman, P., Carpentier, P., Sangalli, M., and Ficarra, V. (2010). Impact of the learning curve on perioperative outcomes in patients who underwent robotic partial nephrectomy for parenchymal renal tumors. Eur. Urol. $58,127-132$.

Naeem, N., Petros, F., Sukumar, S., Patel, M., Bhandari, A., Kaul, S., et al. (2011). Robot-assisted partial nephrectomy in obese patients. $J$. Endourol. 25, 101-105.

Nepple, K. G., Sandhu, G. S., Rogers, C. G., Allaf, M. E., Kaouk, J. H., Figenshau, R. S., et al. (2012). Description of a multicenter safety checklist for intraoperative hemorrhage control while clamped during robotic partial nephrectomy. Patient Saf. Surg. 6, 8. 
Patel, M. N., Krane, L. S., Bhandari, A., Laungani, R. G., Shrivastava, A., Siddiqui, S. A., et al. (2010). Robotic partial nephrectomy for renal tumors larger than $4 \mathrm{~cm}$. Eur. Urol. 57, 310-316.

Petros, F. G., Patel, M. N., Kheterpal, E., Siddiqui, S., Ross, J., Bhandari, A., et al. (2011). Robotic partial nephrectomy in the setting of prior abdominal surgery. BJU Int. 108, 413-419.

Rogers, C. G. (2009). Retroperitoneal Robotic Kidney Surgery: Technique and Early Results. Available at: http:// www.vidoevo.com/yvideo.php?i= alhMUzZCcWuRpZkI4TTQ\& retroperitoneal-robotic-kidneysurgery-technique-and-early-results

Rogers, C. G., Metwalli, A., Blatt, A. M., Bratslavsky, G., Menon, M., Linehan, W. M., et al. (2008). Robotic partial nephrectomy for renal hilar tumors: a multi-institutional analysis. J. Urol. 180, 2353-2356.

Sammon, J., Petros, F., Sukumar, S., Bhandari, A., Kaul, S., Menon, M., et al. (2011). Barbed suture for renorrhaphy during robot-assisted partial nephrectomy. J. Endourol. 25, 529-533.

San Francisco, I. F., Sweeney, M. C., and Wagner, A. A. (2011). Robotassisted partial nephrectomy: early unclamping technique. J. Endourol. 25, 305-308.

Scoll, B. J., Uzzo, R. G., Chen, D. Y., Boorjian, S. A., Kutikov, A., Manley, B. J., et al. (2010). Robot-assisted partial nephrectomy: a large singleinstitutional experience. Urology 75, 1328-1334.
Simhan, J., Smaldone, M. C., Tsai, K. J., Reyes, J. M., Canter, D., Kutikov, A., et al. (2012). Perioperative outcomes of robotic and open partial nephrectomy for moderately and highly complex renal lesions. $J$. Urol. 187, 2000-2004.

Sukumar, S., and Rogers, C. G. (2011) Robotic partial nephrectomy: surgical technique. BJU Int. 108, 942-947. Tanagho, Y. S., Bhayani, S. B., Kim, E. H., Sandhu, G. S., Vaughn, N. P., and Figenshau, R. S. (2012a). Off-clamp robot-assisted partial nephrectomy: initial Washington University experience. J. Endourol. 26, 1284-1289.

Tanagho, Y. S., Bhayani, S. B., Sandhu, G. S., Vaughn, N. P., Nepple, K. G., and Figenshau, R. S. (2012b). Renal functional and perioperative outcomes of off-clamp versus clamped robot-assisted partial nephrectomy: matched cohort study. Urology 80 838-844.

Tanagho, Y. S., Kaouk, J. H., Allaf, M. E., Rogers, C. G., Stifelman, M. D., Kaczmarek, B. F., et al. (2012c). Perioperative complications of robot-assisted partial nephrectomy: analysis of 886 cases at five U.S. centers. Urology (in press).

Thompson, R. H., Boorjian, S. A., Lohse, C. M., Leibovich, B. C., Kwon, E. D., Cheville, J. C., et al. (2008). Radical nephrectomy for pTla renal masses may be associated with decreased overall survival compared with partial nephrectomy. J. Urol. 179, 468-471.

Thompson, R. H., Kaag, M., Vickers, A., Kundu, S., Bernstein, M., Lowrance, W., et al. (2009). Contemporary use of partial nephrectomy at a tertiary care center in the United States. Urol. 181, 993-997.

Thompson, R. H., Lane, B. R., Lohse, C. M., Leibovich, B. C., Fergany, A., Frank, I., et al. (2010). Every minute counts when the renal hilum is clamped during partial nephrectomy. Eur. Urol. 58, 340-345.

Wang, A. J., and Bhayani, S. B. (2008). Robotic partial nephrectomy versus laparoscopic partial nephrectomy for renal cell carcinoma: single-surgeon analysis of $>100$ consecutive procedures. Urology 73, 306-310.

Weight, C. J., Lieser, G., Larson, B. T., Gao, T., Lane, B. R., Campbell, S. C., et al. (2010). Partial nephrectomy is associated with improved overall survival compared to radical nephrectomy in patients with unanticipated benign renal tumors. Eur. Urol. 58, 293-298.

Weizer, A. Z., Palella, G. V., Montgomery, J. S., Miller, D. C., and Hafez, K. S. (2011). Robot-assisted retroperitoneal partial nephrectomy: technique and perioperative results. J. Endourol. 25, 553-557.

White, M. A., Autorino, R., Spana, G., Laydner, H., Hillyer, S. P., Khanna, R., et al. (2011). Robotic laparoendoscopic single-site radical nephrectomy: surgical technique and comparative outcomes. Eur. Urol. 59, 815-822.

Williams, S. B., Kacker, R., Alemozaffar, M., Francisco, I. S., Mechaber, J., and Wagner, A. A. (2011). Robotic partial nephrectomy versus laparoscopic partial nephrectomy: a single laparoscopic trained surgeon experience in the development of a robotic partial nephrectomy program. World J. Urol. doi: 10.1007/s00345-011-06485 [Epub ahead of print].

Wu, S. D., Viprakasit, D. P., Cashy, J., Smith, N. D., Perry, K. T., and Nadler, R. B. (2010). Radiofrequency ablation-assisted robotic laparoscopic partial nephrectomy without renal hilar vessel clamping versus laparoscopic partial nephrectomy: a comparison of perioperative outcomes. J. Endourol. 24, 385-391.

Conflict of Interest Statement: Sam B. Bhayani is a consultant for Surgi Quest, Inc. Youssef S. Tanagho and Robert S. Figenshau have no conflicts of interest.

Received: 02 November 2012; paper pending published: 26 November 2012; accepted: 21 December 2012; published online: 11 January 2013.

Citation: Tanagho YS, Bhayani SB and Figenshau RS (2013) Robot-assisted partial nephrectomy in contemporary practice. Front. Oncol. 2:213. doi: 10.3389/fonc.2012.00213

This article was submitted to Frontiers in Genitourinary Oncology, a specialty of Frontiers in Oncology.

Copyright (C) 2013 Tanagho, Bhayani and Figenshau. This is an open-access article distributed under the terms of the Creative Commons Attribution License, which permits use, distribution and reproduction in other forums, provided the original authors and source are credited and subject to any copyright notices concerning any third-party graphics etc. 